\title{
Modèle à hasard proportionnel pour la fiabilité des composants mécaniques
}

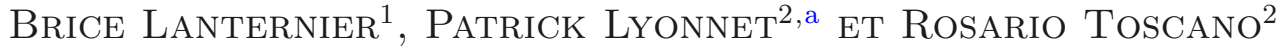 \\ 1 INERIS, Parc ALATA, 60550 Verneuil-en-Halatte, France \\ 2 LTDS ENISE UMR 5513, 58 rue Jean Parot, 42000 St-Étienne, France
}

Reçu le 19 mars 2008, accepté le 9 octobre 2008

\begin{abstract}
Résumé - La fiabilité des matériels mécaniques est largement conditionnée par les caractéristiques propres à chaque système, à ses conditions d'utilisation, son environnement... De plus, les mécanismes de dégradations comme la fatigue et le stress créent des phénomènes de vieillissement. Les bases de données de fiabilité, en fournissant des taux de défaillance génériques et constants, ne permettent pas de prendre en considération ces particularités. L'utilisation de telles bases entraîne par conséquent de grandes incertitudes quant aux résultats des évaluations fiabilistes. Nous proposons dans cet article une modélisation des taux de défaillance, fonction du temps, qui prend en compte les facteurs d'influence. Une application permet de valider le modèle.
\end{abstract}

Mots clés : Taux de défaillance / système mécanique / facteur d'influence / modèle de Cox / distribution de Weibull

\begin{abstract}
Mechanical component reliability, hazard proportionnal model. Mechanical systems reliability depends on design, use, physical environment... Moreover, failure mechanisms as fatigue or stress yield systems ageing. The reliability databases are not allowed to deal with these particularities because of generic and constant failure rates. Many uncertainties in reliability predictions can be due to the use of these databases. A failure rate modelling is introduced in this article, and aims at tacking into account both time and influencing factors. An example describes the methodology and is used for assessing the model accuracy.
\end{abstract}

Key words: Failure rate / mechanical system / influencing factor / Cox modelling / Weibull distribution

\section{Introduction}

Les évaluations fiabilistes des systèmes mécaniques reposent sur de nombreux paramètres dont en premiers lieux les taux de défaillances. Par défaut, des bases de données de fiabilité sont communément utilisées. Pour la plupart, ce sont des recueils de données : OREDA [1], EIReDA [2], exida [3], NPRD-95 [4], PDS Data Handbook [1] et bien d'autres, issus des retours d'expérience de secteurs d'activité variés. Les utilisateurs potentiels de celles-ci, se basent sur le fait que leurs matériels sont sensiblement similaires et que les données de fiabilité de ces bases peuvent être transposées à leurs préoccupations. Or on constate que les données de fiabilité des systèmes étudiés ne sont pas homogènes, comme

\footnotetext{
a Auteur pour correspondance :
} patrick.lyonnet@vanadoo.fr le laissent supposer les variations parfois non négligeables des taux de défaillance entre les bases. Les causes sont nombreuses :

- les matériels ont des caractéristiques qui leur sont propres. Un même type de système se décline sous de nombreux équipements dont la fiabilité est différente ;

- les conditions d'utilisation et l'environnement de fonctionnement varient entre les systèmes. La fiabilité des matériels mécaniques est sensible aux taux de charge, aux modes de fonctionnement, aux sollicitations, aux modes de défaillance pris en considération, aux politiques de maintenance... Ces différences sont accentuées entre les secteurs d'activité;

- faire la synthèse des données recueillies pour chaque type de système, nécessite souvent de regrouper sans distinction des matériels aux propriétés intrinsèques et extrinsèques variées, sans distinction de caractéristiques. 
L'utilisation de ces bases comme données d'entrées des évaluations de fiabilité entraîne par conséquent de grandes incertitudes quant à la pertinence des résultats.

Le second point qu'il convient de noter est que toutes les bases de données exposées ci-dessus ne fournissent que des taux de défaillance constants. Toutefois, les mécanismes de dégradations des composants mécaniques comme par exemple la fatigue, les vibrations, la corrosion et autres stress créent des phénomènes d'usure et par conséquent le système vieillit. À cela on peut ajouter une période de rodage qui provoque généralement des défaillances chez les jeunes systèmes. Tout cela amène les taux de défaillance des systèmes mécaniques à dépendre du temps. Bien qu'elles y fassent allusion (OREDA, [1]), les bases de données n'incluent pas la variable temps.

Prenant en considération ces remarques, nous proposons dans cet article une méthode de modélisation des taux de défaillance, spécialement conçue pour répondre aux caractéristiques des systèmes mécaniques. Les taux de défaillance ainsi modélisés dépendront de ce que nous nommerons les « facteurs d'influence », et seront des fonctions du temps. La seconde partie de cet article présente la notion de «facteurs d'influence ». Le développement d'un modèle sera ensuite proposé dans la troisième partie puis une application illustrera la méthodologie.

\section{Les facteurs d'influence et la fiabilité}

\subsection{Présentation des facteurs d'influence}

\subsubsection{Définition générale et sélection des facteurs d'influence}

Les facteurs d'influence sont des éléments, internes ou externes, qui ont pour effet d'agir sur la fiabilité du système. L'influence peut être positive en provoquant des réductions du nombre de défaillances, ou au contraire, avoir des effets négatifs sur la fiabilité. Le terme est large et peut regrouper une quantité importante de facteurs. Pour répondre à notre besoin de modélisation des taux de défaillance, nous choisirons de prendre en compte un nombre limité de facteurs. La sélection se fait selon trois critères :

- il est possible de mesurer ou évaluer l'état des facteurs pour chaque système;

- les mesures ou évaluations des facteurs doivent permettre de faire des distinctions entre les systèmes étudiés ;

- les facteurs d'influence retenus sont suffisamment exhaustifs pour justifier à eux seuls les différences significatives en matière de fiabilité.

\subsubsection{Classification des facteurs d'influence}

Les références étudiées ne font pas allusion aux mêmes facteurs d'influence pour mener à bien des évaluations fiabilistes. Selon la nature du système étudié (humain, électrique/électronique, mécanique), les facteurs observés ne sont généralement pas les mêmes (facteurs humains ou organisationnels, facteurs intrinsèques ou extrinsèques au système). Après une étude sur les ouvrages et les méthodes consacrées aux facteurs d'influence, nous proposons une classification de ces derniers selon les phases de vie du système étudié :

- les facteurs de conception, de nature intrinsèque comme par exemple le type du système, le principe de fonctionnement, les dimensions ou les matériaux utilisés ;

- les facteurs liés à la fabrication, le fabricant ou le procédé de fabrication;

- les facteurs dus à l'installation du système, comme les techniques et les procédures utilisées dans ce but;

- les facteurs qui influent lors de l'utilisation du système, ce sont ceux relatifs à la fréquence et à la charge de sollicitation, aux exigences en terme de fiabilité, à l'environnement dans lequel le système évolue;

- les facteurs de maintenance, comme par exemple la fréquence des tests préventifs, la quantité et la qualité des autres actions préventives et/ou correctives.

On peut ajouter à cette liste les facteurs humains et organisationnels qui, de part leurs natures, ont une influence généralement plus globale sur le système. Aven et al. [5] proposent une classification de ce type de facteurs. Dans la suite de l'article nous n'évoquerons pas ces facteurs d'influence.

\subsection{Les modèles pour la prise en compte des facteurs d'influence dans la fiabilité}

\subsubsection{Les facteurs d'influence et les systèmes électriques/électroniques}

L'évaluation des taux de défaillance pour les composants électriques/électroniques a donné lieu à des normes dès les années 1960 avec la MIL-HDBK-217 [6]. Ce standard est maintenant bien connu et utilisé dans la défense comme dans l'industrie. Pour un grand nombre de composants référencés, des taux de défaillance, constants dans le temps, sont exprimés analytiquement en fonction de paramètres définis. On note par exemple l'influence de la température, de la tension ou de l'intensité électrique. Fondées sur le même modèle, d'autres références ont vu le jour pour répondre à des domaines précis. Dans le milieu militaire il existe la méthodologie PRISM développée pour l'US Air Force [7] et une norme chinoise, la GJB/z 299B. Pour les télécommunications, Telcordia, ancien Bellcore [8] est la plus répandue mais on peut également trouver la base RDF [9] et la base HRD5.

\subsubsection{Les facteurs d'influence et les systèmes mécaniques}

L'US Navy [10] propose des modèles sur le même principe que la MIL-HDBK-217 mais qui concernent des 
systèmes mécaniques comme des vannes, ressorts, joints, engrenages... Des coefficients d'influence sont calculés à l'aide de relations physiques, fonctions de nombreux paramètres comme la température, la pression, des dimensions, les propriétés des fluides et des matériaux etc. À chaque facteur d'influence est attribué un coefficient qui est ensuite multiplié à des taux de défaillance de base. Le taux de défaillance total d'un système, supposé constant, est exprimé par la somme des taux de défaillance de chacun de ses composants.

Sans connaissance a priori sur les relations physiques qui lient les facteurs d'influence aux taux de défaillance, des méthodes statistiques tentent également d'exprimer des coefficients de correction. L'objectif est là aussi d'adapter des taux de défaillance génériques à l'environnement ou aux caractéristiques du système étudié. Le CCPS Guidelines [11] et l'annexe $7 \mathrm{du}$ projet ARAMIS [12] proposent succinctement quelques approches basées sur le retour d'expérience. Seuls des taux de défaillance constants sont là aussi étudiés.

\subsubsection{Proposition d'un modèle pour les systèmes mécaniques}

Nous avons vu en introduction que des taux de défaillance constants n'étaient généralement pas adaptés aux systèmes mécaniques, notamment à cause des phénomènes d'usure et donc de vieillissement. Aucun de ces modèles répertoriés ne permet pourtant de modéliser des taux de défaillance en fonction du temps. L'approche proposée va donc tenter de répondre à cette exigence. Un modèle fondé sur des analyses statistiques sera préféré aux modélisations physiques. L'objectif est de pouvoir réaliser les études fiabilistes sans connaissance a priori des relations souvent complexes qui lient les facteurs d'influence aux taux de défaillance.

\section{Proposition d'un modèle pour la modélisation des taux de défaillance}

\subsection{Modéliser les taux de défaillance en fonction du temps et des facteurs d'influence}

\subsubsection{Modèle général}

L'approche que nous proposons cherche tout particulièrement à modéliser les taux de défaillance des systèmes mécaniques. Pour cela il convient de répondre aux particularités suivantes, développées dans la partie introductive :

- les taux de défaillance des systèmes mécaniques ne sont généralement pas constants, ils doivent donc être exprimés en fonction du temps (pour certaines applications il peut indifféremment s'agir d'une distance ou autre...);

- la prise en compte des facteurs d'influence doit permettre une adéquation du taux de défaillance avec les caractéristiques internes et externes du système.
Nous proposons un modèle semi-paramétrique de la forme :

$$
\lambda(t, Z)=\lambda_{0}(t) g(Z)
$$

avec :

- $\lambda(t, Z)$ le taux de défaillance du système, fonction du temps $t$ et de l'état $Z$ des facteurs d'influence;

- $\lambda_{0}(t)$ le taux de défaillance de base du système, fonction uniquement du temps;

- $g(Z)$ une fonction que l'on appellera fonction d'influence et qui dépend uniquement de l'état des facteurs d'influence.

\subsubsection{Taux de défaillance de base $\lambda_{0}(t)$}

Nous avons vu précédemment que les matériels mécaniques répondaient rarement à un taux de défaillance constant, synonyme d'une distribution de probabilité exponentielle. Ici nous proposerons une modélisation par la loi de Weibull à deux paramètres. Le taux de défaillance de base s'exprime alors ainsi :

$$
\lambda_{0}(t)=\frac{\beta}{\eta}\left(\frac{t}{\eta}\right)^{\beta-1}
$$

avec :

- $\beta$ le paramètre de forme, sans unité ;

- $\eta$ le paramètre d'échelle en unité de temps. On trouve parfois l'expression (2) sous une forme qui pose le paramètre $\lambda=1 / \eta$.

Cette distribution est largement répandue dans la fiabilité des systèmes mécaniques car elle permet de modéliser trois périodes de vie du système selon la valeur du paramètre $\beta$ :

- une période de rodage, dite aussi de mortalité infantile, si $\beta<1$. Le taux de défaillance est décroissant en fonction du temps. Les défaillances qui apparaissent dans cette première phase de vie sont classiquement dues à des défauts de conception ou d'installation découverts qu'une fois le système mis en marche. La plupart de ces défaillances peuvent être évitées par des politiques de tests, avant la mise en place du matériel ([13], p. 21);

- une phase dite de vie utile, si $\beta \approx 1$. Le taux de défaillance est alors quasi-constant. Les défauts matériels écartés, le système est dans sa phase de vie principale. Les défaillances qui surviennent durant cette période ont pour cause des évènements aléatoires indépendants du temps et de l'âge du système;

- une phase d'usure, si $\beta>1$. Le taux de défaillance est croissant en fonction du temps. Le stress, la fatigue, la corrosion etc. détériorent le système, ce qui accentue la probabilité qu'une défaillance ait lieu durant cette période.

L'allure du taux de défaillance ainsi représenté durant ces trois phases de vie, lui donne le nom de « courbe 
en baignoire ». Les bases de données ne fournissent généralement que des taux de défaillance de vie utile, supposés constants. D'autres distributions comme la loi Gamma, Normale, Log-Normale, Birnbaum-Saunders, Gaussienne inverse et autres peuvent aussi être envisagées dans le modèle.

\subsubsection{Fonction d'influence $g(Z)$}

La fonction d'influence sera indépendante du temps. Cela implique certaines hypothèses comme le fait que les distributions des temps de vie du système doivent appartenir aux mêmes familles paramétriques, quels que soient les états $Z$ des facteurs d'influence. Plusieurs facteurs et des plus variés pouvant entrer en jeu, $Z$ sera un vecteur dont les composantes $\left\{z_{k}\right\}_{k=1}, \ldots, m$ seront indifféremment de natures binaires, discrètes ou continues. La partie sur le codage détaillera ce point. Le choix d'un modèle de Cox pour l'expression de la fonction d'influence est motivé par :

- sa particularité d'être « libre de distribution », c'està-dire qu'il peut être associé dans la relation (1) à n'importe quelle distribution [14] ;

- la non-nécessité de connaître les relations a priori qui lient les facteurs d'influence à fiabilité du système (le modèle est purement statistique);

- les caractéristiques de codage qui permettent d'utiliser des paramètres quantitatifs, qualitatifs, une combinaison des deux, ainsi que des valeurs imprécises;

- la capacité à comparer le sens et le degré d'influence de chaque facteur par l'intermédiaire des coefficients $\left\{b_{k}\right\}_{k=1}, \ldots, m$ développés ci-après;

- la prédisposition à prendre en compte les interactions entre les facteurs d'influence [15];

- la possibilité d'exprimer simplement le rapport entre deux taux de défaillance, correspondant à des configurations distinctes d'états des facteurs d'influence;

- la compétence, sous certaines conditions, d'évaluer des taux de défaillance de systèmes présents dans des associations inédites de facteurs d'influence.

Le modèle de Cox est déjà proposé pour l'étude de la fiabilité sous vieillissement accéléré [16], et est bien répandu dans les applications biomédicales [14]. D'autres modélisations comme par exemple l'utilisation des réseaux de neurones peuvent être envisagées. Ces derniers ont néanmoins l'inconvénient d'agir comme une «boîte noire » [17] et n'ont pas tous les avantages du modèle de Cox cités précédemment. Nous proposons donc une fonction d'influence sous une des formes proposées par Cox [18] :

$$
g(Z)=\mathrm{e}^{B Z}=\mathrm{e}^{\sum_{k=1}^{m} b_{k} z_{k}}
$$

avec :

- $m$ le nombre de facteurs d'influence pris en compte dans le modèle;

- $B=\left[b_{1}, \ldots, b_{m}\right]$ le vecteur des coefficients du modèle de Cox;
$-Z=\left[z_{1}, \ldots, z_{m}\right]$ le vecteur des états des facteurs d'influence.

\subsection{Construction du modèle proposé}

\subsubsection{Résumé des étapes}

La construction du modèle que nous proposons se fait en cinq étapes :

- recueil des données d'entrées, à savoir les temps de bon fonctionnement avant défaillance ainsi que les états des facteurs d'influence associés, et pour chacun des systèmes observés :

- établissement d'un codage pour les états des facteurs d'influence ;

- détermination des paramètres de la fonction d'influence;

- détermination des paramètres du taux de défaillance de base ;

- synthèse des résultats.

Remarque : le choix des facteurs d'influence et des indicateurs caractérisant leurs états ne seront pas développés dans cet article.

\subsubsection{Recueil des données d'entrées}

La première étape consiste à collecter les durées de bon fonctionnement des matériels avant leurs défaillances: Time to Failure (TtF). On notera ces temps $T_{i}$ avec $i=1, \ldots, n$ où $n$ est le nombre de données recueillies. Dans un premier temps nous supposerons que ces données ne sont pas censurées. Pour des systèmes réparables nous disposons généralement de plusieurs TtF par matériel. Un même système peut alors se voir associer plusieurs indices $i$.

Enfin, pour chaque relevée $i$ il convient également de recueillir les états des facteurs d'influence correspondants.

\subsection{3 Établissement d'un codage pour les états des facteurs d'influence}

Afin d'être utilisables dans une modélisation de Cox [18], les états des facteurs d'influence doivent être codés. Pour cela il existe plusieurs possibilités :

- pour les facteurs quantitatifs (pression en bar, température en degrés Celsius, diamètre en cm...) les valeurs de mesures peuvent être utilisées en l'état;

- pour les facteurs qualitatifs il est possible de définir des valeurs discrètes associées à des « classes ». Par exemple un type de matériel est codé par la valeur 1 , un second type par la valeur 2. Le même principe peut être utilisé pour les origines de fabrication, les matériaux utilisés etc.; 
- pour rendre les évaluations plus flexibles, les valeurs continues peuvent être discrétisées en définissant des intervalles, disjoints ou non. Par exemple nous codons avec la valeur 1 tous les systèmes soumis à une température inférieure à $10^{\circ} \mathrm{C}$, par 2 lorsque la température est comprise entre $10{ }^{\circ} \mathrm{C}$ et $50{ }^{\circ} \mathrm{C}$, et par 3 ceux dépassant les $50^{\circ} \mathrm{C}$. Ainsi on peut faire face à deux réalités pratiques :

- les mesures sont toujours soumises à un certain degré d'incertitude;

- les états des facteurs d'influence peuvent varier dans le temps;

Il est tout à fait possible de combiner ces différents codages dans un même modèle. On notera $Z_{i}$ le vecteur des états $\left\{z_{k}\right\}_{k}=1, \ldots, m$ des facteurs d'influence associés au relevé d'indice $i$.

\subsubsection{Détermination des paramètres de la fonction d'influence}

L'objectif est de déterminer le vecteur $B=\left[b_{1}, \ldots\right.$, $b_{m}$ ] des coefficients du modèle de Cox [18], en utilisant les relevés $T_{i}$ et les vecteurs d'états $Z_{i}$ associés. Nous préconisons pour cela le maximum de vraisemblance de Cox. Rappelons que la vraisemblance $V^{*}$ est égale au produit des contributions $V_{i}[17]$ telles que :

$$
\begin{aligned}
V^{*} & =\prod_{i=1}^{n} V_{i}=\prod_{i=1}^{n} \frac{\lambda\left(T_{i}, Z_{i}\right)}{\sum_{j \in R_{i}} \lambda\left(T_{j}, Z_{j}\right)} \\
& =\prod_{i=1}^{n} \frac{\mathrm{e}^{B Z_{i}}}{\sum_{j \in R_{i}} \mathrm{e}^{B Z_{j}}}
\end{aligned}
$$

avec :

- $n$ le nombre de recueils pour les données d'entrées;

- $R_{i}$ l'ensemble des indices correspondant à des systèmes encore en fonctionnement à l'instant $T_{i}$, l'indice $i$ inclus ; c'est-à-dire l'ensemble des indices $j$ tels que $T_{j} \leq T_{i}$.

En passant l'expression (4) sous sa forme logarithmique, on cherchera le vecteur $B$ qui maximise $L^{*}$ tel que [16] :

$$
L^{*}(B)=\sum_{i=1}^{n}\left(B Z_{i}-\ln \left(\sum_{j \in R_{i}} \mathrm{e}^{B Z_{j}}\right)\right)
$$

Des méthodes numériques permettent d'obtenir la solution à ce problème d'optimisation. Un test simple permet d'évaluer le degré de confiance de l'estimation [16] :

$$
\chi_{m}^{2}=-2\left[L^{*}(0)-L^{*}(B)\right]
$$

avec :

- $m$ la dimension du vecteur $B$;

- 0 le vecteur nul de dimension $m$;

- $\chi_{m}^{2}$ qui suit une loi du Chi ${ }^{2}$ à $m$ degrés de liberté.
Le degré de confiance se mesure alors par la probabilité réciproque de la valeur du $\mathrm{Chi}^{2}$ ainsi obtenue. Une faible probabilité indique un bon degré de confiance.

À cette étape il est déjà possible de calculer les rapports $C\left(Z_{1}, Z_{2}\right)$ entre les taux de défaillance de deux états $Z_{1}$ et $Z_{2}$ des facteurs d'influence :

$$
C\left(Z_{1}, Z_{2}\right)=\frac{\lambda\left(t, Z_{2}\right)}{\lambda\left(t, Z_{1}\right)}=\frac{\lambda_{0}(t) \mathrm{e}^{B Z_{2}}}{\lambda_{0}(t) \mathrm{e}^{B Z_{1}}}=\mathrm{e}^{B\left(Z_{2}-Z_{1}\right)}
$$

\subsubsection{Détermination des paramètres du taux de défaillance de base}

La fonction d'influence $g(Z)$ étant définie, nous cherchons maintenant à exprimer le taux de défaillance de base $\lambda_{0}(t)$. Dans un premier temps nous proposons d'exprimer les TtF théoriques de base que l'on notera $T_{0, i}$. Il s'agit des TtF que l'on aurait « théoriquement » recueilli si les fonctions d'influence $g\left(Z_{i}\right)$ n'étaient pas entrées en jeu. Il est important de signaler que compte tenu de la détermination de la fonction $g(Z)$, les TtF théoriques de base n'ont aucune signification physique. Rappelons que si les TtF suivent une distribution de Weibull dont le taux de défaillance $\lambda_{0}(t)$ est exprimé par l'équation (2), une multiplication de ce taux par le coefficient $g\left(Z_{i}\right)$ entraîne théoriquement une division des TfF par $g\left(Z_{i}\right)^{1 / \beta}$. Nous obtenons alors les relations suivantes:

$$
T_{i}=\frac{T_{0, i}}{\left(g\left(Z_{i}\right)\right)^{\frac{1}{\beta}}} \Leftrightarrow T_{0, i}=T_{i}\left(g\left(Z_{i}\right)\right)^{\frac{1}{\beta}}
$$

On remarque que les $T_{0, i}$ dépendent du paramètre $\beta$, d'où la problématique pour estimer directement les paramètres $\beta$ et $\eta$ en utilisant ces $T_{0, i}$ dans une approche classique comme la méthode graphique ou de régression. Nous proposons alors une estimation par le maximum de vraisemblance. L'obtention du paramètre $\beta$ peut alors se faire par la résolution numérique de l'égalité suivante [18] :

$$
\frac{n}{\beta}+\sum_{i=1}^{n} \ln \left(T_{0, i}\right)-\frac{n \sum_{i=1}^{n}\left(T_{0, i}^{\beta} \ln \left(T_{0, i}\right)\right)}{\sum_{i=1}^{n} T_{0, i}^{\beta}}=0
$$

Connaissant les $T_{i}$, l'inclusion de l'expression (8) dans l'égalité (9) permet d'obtenir une équation à une seule inconnue : $\beta$. Le paramètre $\eta$ se calcule ensuite directement $\operatorname{par}[13]$ :

$$
\eta=\left(\frac{\sum_{i=1}^{n} T_{0, i}^{\beta}}{n}\right)^{1 / \beta}
$$

Rausand et al. ([13], p. 510) proposent également certaines expressions pour prendre en compte des données censurées.

\subsubsection{Synthèse des résultats}

À cette étape nous disposons de toutes les informations nécessaires à l'expression du taux de défaillance, en 
fonction du temps $t$ et de l'état des facteurs d'influence $Z$ :

$$
\lambda(t, Z)=\lambda_{0}(t) g(Z)=\frac{\beta}{\eta}\left(\frac{t}{\eta}\right)^{\beta-1} \mathrm{e}^{B Z}
$$

\section{Application du modèle proposé et discussion des résultats}

\subsection{Application du modèle proposé}

\subsubsection{Présentation de l'exemple}

Pour appliquer le modèle que nous proposons et être en mesure de l'évaluer par la suite, nous allons utiliser une étude de cas fictive sur des soupapes de sécurité. 56 matériels sont étudiés et 3 facteurs d'influence sont pris en compte :

- la pression nominale d'activation de la soupape. Nous distinguons trois classes de matériels : 7 bars, 20 bars et 70 bars. Il ne s'agit ici que de pressions nominales, les valeurs réelles étant difficiles à évaluer avec précision ;

- les dimensions de la soupape. On dispose de petites soupapes (que l'on appellera de catégorie A) pour des niveaux de pression allant jusqu'à 20 bars, des soupapes de taille moyenne (catégorie B) pour tous types de pression, et des soupapes plus grandes (catégorie C) pour des pressions supérieures à 20 bars;

- suivant la dangerosité du produit retenu par les soupapes, on définit deux niveaux de tolérance aux fuites externes de celles-ci : restreint et indulgent. Bien évidemment, les soupapes pour lesquels le niveau de tolérance aux fuites est restreint sont plus souvent considérées comme défaillantes que les autres.

Nous utiliserons comme référence, des taux de défaillance théoriques. Ceux-ci se basent sur une loi de Weibull avec un paramètre de forme $\beta_{\text {th }}=1,7$ et un paramètre d'échelle $\eta_{\mathrm{th}}=18000 \mathrm{~h}$. Des coefficients d'influence ont ensuite été calculés en s'appuyant sur des formules de la NSWC-98/LE1 [10]. Utilisant ces paramètres, des temps de bon fonctionnement avant défaillance ont été simulés par la méthode de Monte Carlo. Ces TtF serviront de données d'entrées à notre application. Les cinq étapes du modèle sont décrites ci-après.

\subsubsection{Recueil des données d'entrées}

Les soupapes sont numérotées de 1 à 56 . Elles sont regroupées par quatre, suivant les configurations nominales des facteurs d'influence. La répartition des soupapes est résumée dans le tableau 1 . Il convient de noter qu'il ne s'agit que de configurations nominales. Ainsi, et même parmi les groupes de quatre, chaque soupape a un taux de défaillance qui lui est propre. Les écarts de pression par rapport aux valeurs nominales, justifient notamment le fait que les taux de défaillance soient particuliers à chaque
Tableau 1. Recueil des données, répartition des soupapes par facteur d'influence.

\begin{tabular}{cccc}
\hline $\begin{array}{c}\text { Numéro de } \\
\text { soupape }\end{array}$ & $\begin{array}{c}\text { Pression } \\
\text { nominale }\end{array}$ & $\begin{array}{c}\text { Niveau de fuite } \\
\text { acceptable }\end{array}$ & $\begin{array}{c}\text { Catégorie de } \\
\text { dimensions }\end{array}$ \\
\hline 1 à 4 & 7 bars & restreint & catégorie A \\
5 à 8 & 7 bars & restreint & catégorie B \\
9 à 12 & 7 bars & indulgent & catégorie A \\
13 à 16 & 7 bars & indulgent & catégorie B \\
17 à 20 & 20 bars & restreint & catégorie A \\
21 à 24 & 20 bars & restreint & catégorie B \\
25 à 28 & 20 bars & restreint & catégorie C \\
29 à 32 & 20 bars & indulgent & catégorie A \\
33 à 36 & 20 bars & indulgent & catégorie B \\
37 à 40 & 20 bars & indulgent & catégorie C \\
41 à 44 & 70 bars & restreint & catégorie B \\
45 à 48 & 70 bars & restreint & catégorie C \\
49 à 52 & 70 bars & indulgent & catégorie B \\
53 à 56 & 70 bars & indulgent & catégorie C \\
\hline
\end{tabular}

matériel. Sur la figure 1 les TtF simulés, en heures, sont représentés en tirets. Ce sont les données collectées qui nous serviront d'entrées à notre modèle. À titre d'information, les TtF moyens théoriques sont également présentés, en traits gris.

\subsection{3 Établissement du codage pour les états des facteurs d'influence}

Compte tenu de la situation on choisit de faire un codage de 1 à 3 en valeurs discrètes. Celles-ci sont résumées dans le tableau 2.

\subsubsection{Détermination des paramètres de la fonction d'influence}

Par maximisation du rapport de vraisemblance de l'expression (5) et en utilisant les TtF simulés comme données d'entrées $T_{i}$, nous obtenons les coefficients $\left\{b_{k}\right\}_{k=1,2,3}$ du modèle de Cox, donnés dans le tableau 3 .

Compte tenu de l'approche choisie pour l'expression des taux de défaillance théoriques, utilisés pour la simulation des $\mathrm{TtF}$, la relation (6) nous confirme un très bon degré de confiance dans le modèle de Cox. Il convient de nuancer ce résultat par rapport à une situation moins favorable où par exemple les facteurs d'influence ne seraient pas tous identifiés. Des coefficients obtenus, nous pouvons en déduire que le taux de défaillance croît en fonction de la pression et des dimensions. À l'opposé, le signe négatif du coefficient $b_{2}$ nous informe logiquement qu'une tolérance indulgente aux fuites de la soupape entraîne une réduction du taux de défaillance. 


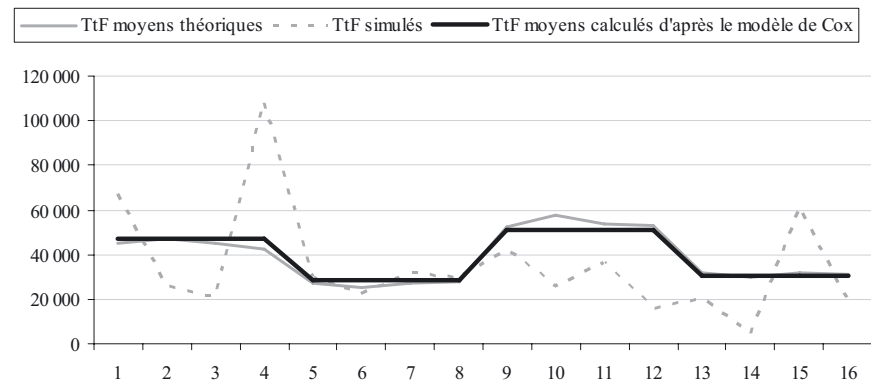

[heures]

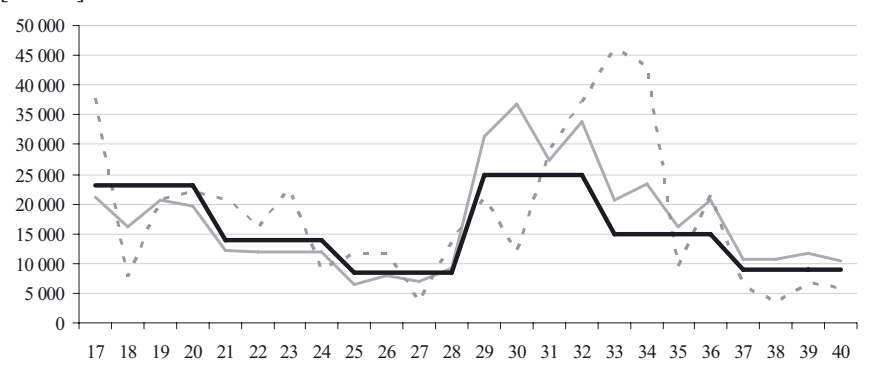
[heures]

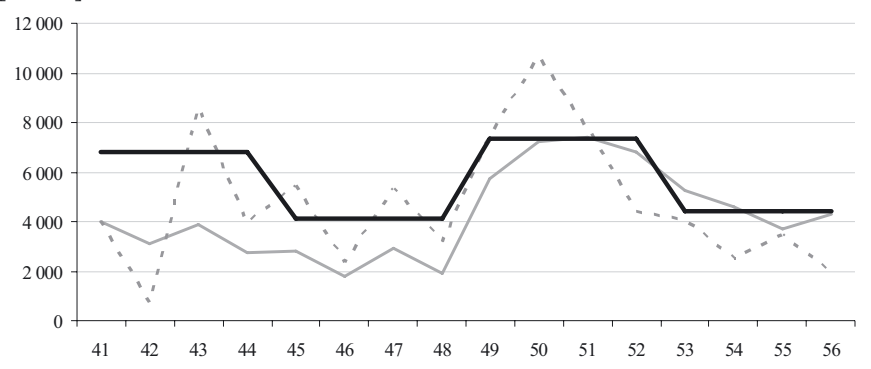

Fig. 1. Représentation des $\mathrm{TtF}$ moyens théoriques, des $\mathrm{TtF}$ simulés, et des TtF moyens calculés d'après le modèle présenté.

D'après la relation (3) nous obtenons alors les fonctions d'influence suivantes :

$$
g\left(Z_{i}\right)=\mathrm{e}^{B Z_{i}}=\mathrm{e}^{1,276 z_{1}-0,140 z_{2}+0,901 z_{3}}
$$

\subsubsection{Détermination des paramètres du taux de défaillance de base}

Par résolution numérique de l'équation (9), puis de l'égalité (10), nous obtenons les paramètres suivants pour l'expression du taux de défaillance de base :

$-\beta=1,784$

$-\eta=165905 \mathrm{~h}$

Soit, d'après la relation (2), le taux de défaillance de base suivant :

$$
\lambda_{0}(t)=\frac{\beta}{\eta}\left(\frac{t}{\eta}\right)^{\beta-1}=\frac{1,784}{165905}\left(\frac{t}{165905}\right)^{0,784}
$$

\subsubsection{Synthèse des résultats}

Par multiplication des expressions (12) et (13) nous modélisons les taux de défaillance des soupapes de
Tableau 2. Codage des facteurs d'influence.

\begin{tabular}{cccc}
\cline { 2 - 4 } & 1 & 2 & 3 \\
\hline Pression nominale & 7 bars & 20 bars & 70 bars \\
Fuite acceptable & restreint & indulgent & - \\
Dimensions & catégorie A & catégorie B & catégorie C \\
\hline
\end{tabular}

Tableau 3. Vecteur $B$ des coefficients du modèle de Cox.

\begin{tabular}{cccc}
\hline Coefficient & $b_{1}$ & $b_{2}$ & $b_{3}$ \\
\hline Valeur & 1,276 & $-0,140$ & 0,901 \\
\hline
\end{tabular}

sécurité de notre application, en fonction du temps $t$ et de l'état des facteurs d'influence $Z$ :

$$
\lambda(t, Z)=\frac{1,784}{165905}\left(\frac{t}{165905}\right)^{0,784} \mathrm{e}^{1,276 z_{1}-0,140 z_{2}+0,901 z_{3}}
$$

Les TtF moyens, estimés d'après la modélisation proposée, sont représentés en traits noirs sur la figure 1. Pour rappel, le temps moyen de bon fonctionnement avant défaillance $(M T T F)$ du composant numéro $i$ s'exprime ici par la relation suivante:

$$
M T T F_{i}=\eta \Gamma\left(1+\frac{1}{\beta}\right)\left(g\left(Z_{i}\right)\right)^{-\frac{1}{\beta}}
$$

avec $\Gamma($.$) la fonction Gamma ([13], p. 573).$

\subsection{Discussion des résultats}

\subsubsection{Discussion sur les paramètres du taux de défaillance de base}

Le paramètre de forme $\beta$ obtenu pour le taux de défaillance de base du modèle $(1,784)$ est proche de celui utilisé pour le taux de défaillance de base théorique $(1,7)$. En effet, l'influence des facteurs sur le taux de défaillance théorique, se fait par l'intermédiaire de coefficients multiplicateurs. Ces coefficients étant indépendants du temps, ils sont assimilables aux fonctions d'influence du modèle présenté. Ces coefficients n'ont donc pas pour effet de modifier l'allure des taux de défaillance en fonction du temps. Il est par conséquent tout à fait adéquat d'obtenir, pour notre modèle, un paramètre de forme proche de l'original.

Le paramètre d'échelle $\eta$ du modèle développé (165905 h) est quant à lui supérieur d'un ordre de grandeur à celui utilisé pour les taux de défaillance de base théoriques $(18000 \mathrm{~h})$. On explique cela par le fait que la détermination des coefficients du modèle de Cox est indépendante des ordres de grandeur des $T_{i}$ utilisés en données d'entrées. Seule la hiérarchie des TtF est prise en 


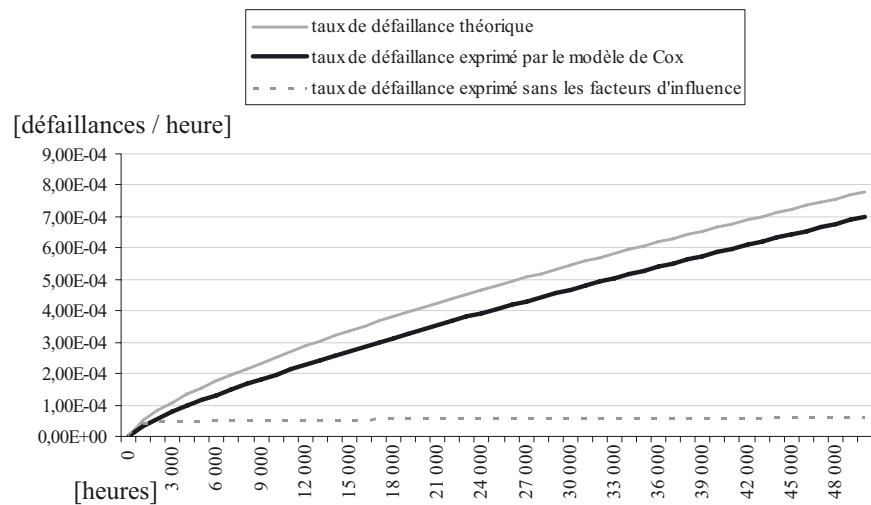

Fig. 2. Représentation du taux de défaillance de la soupape numéro 27.

compte (cf. Éqs. (4) et (5)). Ainsi nous obtenons des fonctions d'influence qui, dans notre exemple, sont d'ordre 10 à $10^{2}$, alors que les coefficients de base, calculés en s'inspirant des formules de la NSWC-98/LE1, étaient d'ordre $10^{-1}$ à 10 . Le paramètre $\eta$ qui est à juste titre un paramètre d'échelle, permet de corriger cet écart.

\subsubsection{Discussion sur les temps moyens de bon fonctionnement avant défaillance}

La figure 1 représente les TtF moyens théoriques (qui ont servi à l'obtention des $\mathrm{TtF}$ simulés) et ceux résultant du modèle que nous proposons. Tout d'abord nous remarquons que les résultats sont cohérents, l'allure générale est respectée. Les valeurs obtenues par le modèle semblent globalement satisfaisantes, elles sont par ailleurs en moyenne plus proches des résultats théoriques que les TtF simulées. On constate que l'adéquation avec les données théoriques dépend notamment de la répartition des temps simulés. Par exemple les TtF simulés pour les soupapes 43 à 48 sont supérieures aux TtF moyens théoriques, le modèle de Cox suit alors une tendance à la surestimation.

\subsubsection{Discussion sur l'expression des taux de défaillance}

Le taux de défaillance de la soupape de sécurité numéroté 27 dans le tableau 1 est représenté sur la figure 2 et la soupape numéro 32 sur la figure 3. Les taux de défaillance théoriques sont reproduit en gris sur chacune des figures, ainsi que les modélisations faites par l'équation (14), en noir. Le vecteur d'états pour les facteurs d'influence est $Z=\left[z_{1}, z_{2}, z_{3}\right]=[2,1,3]$ (resp. [2, $2,1]$ ) pour la soupape numéro 27 (resp. 32), en accord avec le codage établi. Nous avons, de plus, illustré en tirets une modélisation des taux de défaillance qui poserait comme hypothèse l'homogénéité des soupapes, c'està-dire qui ne prend pas en compte les facteurs d'influence. Cette dernière estimation, donnée uniquement à titre de

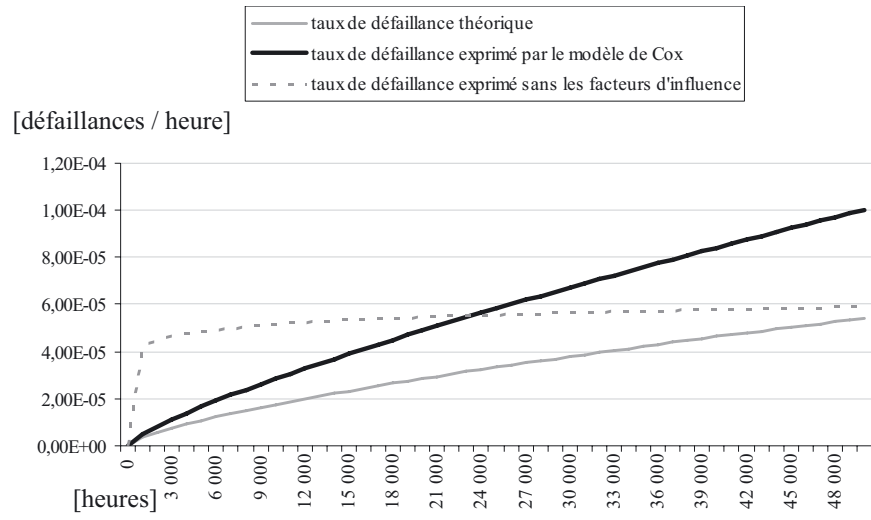

Fig. 3. Représentation du taux de défaillance de la soupape numéro 32.

comparaison, est une loi de Weibull paramétrée directement à l'aide des TtF simulées, elle est donc identique à toutes les soupapes.

Sur la figure 2, nous constatons une très bonne adéquation du taux de défaillance exprimé par notre modèle, avec le taux de défaillance théorique. Une estimation qui ne prendrait pas en compte les facteurs d'influence (représentation en trait gris) impliquerait dans cet exemple une erreur importante et dans ce cas précis une sous-estimation du taux de défaillance. Même s'il ne s'agit que d'un exemple, il est aisé de vérifier que l'adéquation entre les taux de défaillance estimés par le modèle, et les valeurs théoriques, est globalement respectée et ce, pour l'ensemble des soupapes étudiées. Les écarts les plus importants sont observés pour les mêmes configurations où les TtF moyens estimés d'après le modèle sont les plus distants des TtF moyens théoriques (cf. soupapes 29 à 36 et 41 à 48 sur la Fig. 1). Même dans ces cas extrêmes, les estimations qui prennent en compte les facteurs d'influence par le modèle de Cox respectent mieux l'allure et l'ordre de grandeur des taux de défaillance théoriques.

Dans un cas moins favorable comme pour la soupape numéro 32 (Fig. 3), nous observons par exemple une surestimation du taux de défaillance. Par rapport à une modélisation qui ne prendrait pas en compte les facteurs d'influence, le modèle que nous proposons fournit dans un premier temps une meilleure estimation. Ensuite, et même si l'allure n'est pas respectée, la tendance est inversée. Suivant les politiques de maintenance, l'estimation du taux de défaillance d'un matériel dans ses premières phases de vie est souvent la plus importante. Au bout d'une certaine durée de vie, des actions de maintenance préventives sont en effet généralement planifiées.

\section{Conclusion}

Nous avons vu dans cet article que l'utilisation des taux de défaillance génériques comme données d'entrée pour les évaluations fiabilistes des systèmes mécaniques donnaient lieu à de nombreuses incertitudes, notamment 
parce que les bases de données ne fournissent qu'exclusivement :

- des valeurs moyennes, qui ne reflètent pas les disparités parfois importantes entre les systèmes, par exemple à cause des caractéristiques intrinsèques et extrinsèques des matériels ;

- des taux de défaillance constants, qui négligent en particulier les phénomènes d'usure auxquels sont généralement soumis les composants mécaniques.

Nous proposons par conséquent une modélisation des taux de défaillance qui :

- prend en compte les facteurs d'influence auxquels sont soumis les systèmes;

- est fonction du temps.

Pour répondre à ces objectifs, le modèle qui a été présenté dans cet article combine un taux de défaillance de base, dépendant du temps, avec une fonction d'influence, basée sur le modèle de Cox. Dans une démarche générale nous avons proposé une distribution de Weibull pour l'expression du taux de défaillance de base. Celleci a l'avantage d'être à même de modéliser la durée de vie d'un système qui vieillit ou qui est en période de rodage. Il n'est néanmoins pas exclu d'adapter la démarche proposée à n'importe quelle autre distribution.

Le modèle de Cox a quant à lui des avantages pratiques certains. Parmi ceux-ci on note que le modèle est purement statistique et qu'aucune connaissance sur les phénomènes physiques à l'origine de l'influence des facteurs n'est nécessaire. De plus, la souplesse dans le codage permet de faire face aux valeurs quantitatives et/ou qualitatives, imprécises et parfois même variables, dont nous pouvons disposer. Néanmoins, comme toutes exploitations statistiques, l'adéquation du modèle de Cox est fortement liée à la quantité et à la qualité des informations dont nous disposons pour l'étude.

L'application développée a montré comment, dans des conditions favorables en terme de données d'entrées, le modèle proposé permet d'exprimer de façon très adéquate les taux de défaillance des systèmes mécaniques. Sous ces conditions, l'utilisation du modèle permet de mener à bien une évaluation fiabiliste, et ce de façon bien plus précise que l'utilisation conventionnelle des bases de données ou même que d'une analyse statistique qui ne prendrait pas en compte les facteurs d'influence.

Remerciements. Les auteurs tiennent à remercier le Laboratoire d'évaluation des équipements électriques (LEEL) de l'Institut national de l'environnement industriel et des risques (INERIS). Nous remercions également et tout particulièrement le Ministère de l'Écologie et du Développement Durable qui finance nos recherches.

\section{Références}

[1] SINTEF industrial management, Offshore reliability data (OREDA), 4th edition, 2002

[2] H. Procaccia, P. Aufort, S. Arsenis, The European industry reliability data bank (EIReDA), edition, 1998

[3] L.L.C. Exida, Electrical \& Mechanical Component Reliability Handbook, 2005

[4] Reliability Analysis Center (RAC), Nonelectronic Parts Reliability Data 1995 (NPRD-95), 1995

[5] T. Aven, S. Sklet, J.E. Vinnem, Barrier and operational risk analysis of hydrocarbon releases (BORA-Release), J. Hazardous Mat. 137 (2006) 681-691

[6] United States Department of Defense (DoD), MILHDBK-127, Reliability Prediction of Electronic Equipment, 1995

[7] Reliability Analysis Center (RAC) for the US Air Force, PRISM methodology, 1999

[8] Telcordia Technology Inc., Telcordia standard, Reliability Procedure for Electronic Equipment, 2001

[9] Union Technique de l'Électricité (UTE), RDF 2003: Reliability Data Handbook, 2003

[10] Naval Surface Warfare Center (NSWC), NSWC-98/LE1, Handbook of reliability prediction procedures for mechanical equipment, 1998

[11] Center for Chemical Process Safety (CCPS), CCPS guidelines for chemical process quantitative risk analysis, 2nd edition, 2000

[12] B. Debray, E. Piatyszek, F. Cauffet, H. Londiche, ARAMIS DIC, Appendix 7 Frequencies data for the fault tree, 2007

[13] M. Rausand, A. Høyland, System Reliability Theory, Models, Statistical Methods, and applications, Second Edition, New Jersey, Editions Wiley, 2004

[14] K.J. Carroll, On the use and utility of the Weibull model in the analysis of survival data, Controlled clinical trials 24 (2003) 682-701

[15] K. Øien, A framework for establishment of organizational risk indicators, Reliability Eng. System Safety 74 (2001) 147-167

[16] J.C. Augé, J. Lallement, P. Lyonnet, Reliability of mechanical components accelerated testing and advanced statistical methods, Adv. Safety Reliability: ESREL'97, Vol. 3, Lisbon, 17-20 June 1997, C. G. Soares (éd.), pp. $1859-1868$

[17] P. Lyonnet, R. Toscano, P.L. Maisonneuve, B. Lanternier, Modélisation de la fiabilité des macro-composants mécaniques à partir de modèles mathématiques et de réseaux de neurones, comparaisons des méthodes, Proceedings of 24 th Machinery Vibration, Reliability and Maintenance, 25-27 octobre 2006, Montréal, CMVA

[18] D.R. Cox, Regression models and life tables, J. Royal Statistical Soc., series B 34 (1972) 187-220

[19] SINTEF, Reliability data for safety instrumented systems, PDS data handbook, 2006 